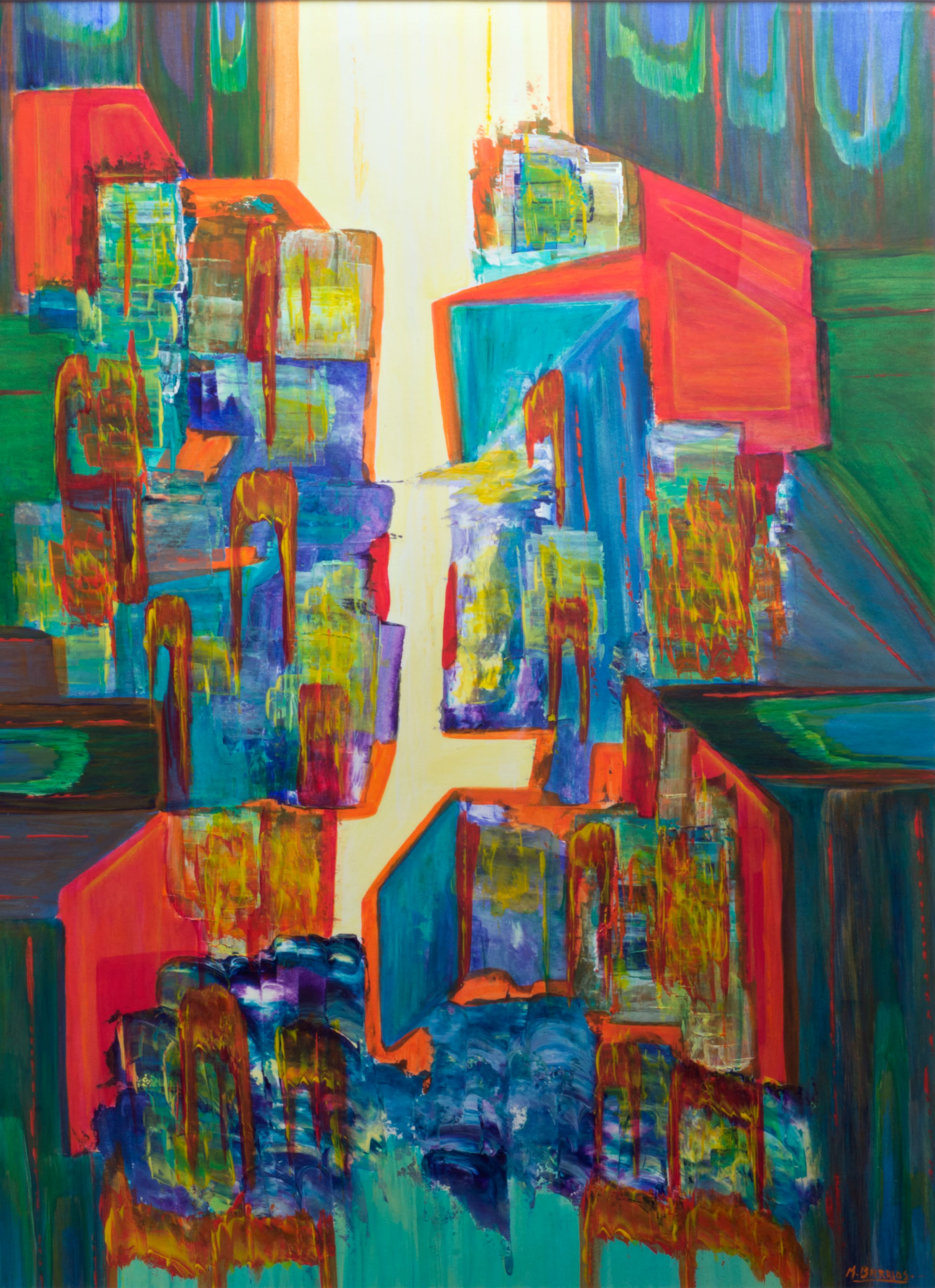




\section{cNuere lentamente}

Muere lentamente quien no viaja,

quien no lee,

quien no oye música,

quien no encuentra gracia en sí mismo.

Muere lentamente

quien destruye su amor propio,

quien no se deja ayudar.

Muere lentamente

quien se transforma en esclavo del hábito

repitiendo todos los días los mismos

trayectos,

quien no cambia de marca,

no se atreve a cambiar el color de su

vestimenta

o bien no conversa con quien no

conoce.

Muere lentamente

quien evita una pasión y su remolino

de emociones,

justamente estas que regresan el brillo

a los ojos y restauran los corazones

destrozados.

Muere lentamente

quien no gira el volante cuando esta infeliz con su trabajo, o su amor,

quien no arriesga lo cierto ni lo incierto para ir detrás de un sueño

quien no se permite, ni siquiera una vez en su vida,

huir de los consejos sensatos... ¡Vive hoy!

iArriesga hoy!

¡Hazlo hoy!

¡No te dejes morir lentamente!

¡No te impidas ser feliz

$\operatorname{coc} c$

Pablo Neruda 


\title{
2. Bienestar subjetivo y su relación con el ingreso económico absoluto, el bienestar objetivo y la condición laboral, en habitantes del municipio de Tatumbla, Francisco Morazán, 2016.
}

Miguel Landa Blanco ${ }^{1}$

Recibido 12-12-2016 / Aceptado 24-02-2017

\begin{abstract}
RESUMEN: La presente investigación tuvo como objetivo analizar el bienestar subjetivo, comprendido como satisfacción con la vida, en habitantes del casco urbano del municipio de Tatumbla, Francisco Morazán en Honduras. Temporalmente, el estudio se realizó en el primer semestre del año 2016. De manera particular, se estudió tal constructo en relación a variables tales como el ingreso económico, el bienestar objetivo y la condición laboral.Todo ello, por medio de un enfoque cuantitativo, con un diseño no experimental, de alcance relacional, en una temporalidad transversal. Para la recolección de información se elaboró una escala compuesta por tres dimensiones: egocéntrica, sociocéntrica y homeostática.
\end{abstract}

Los resultados de la investigación indican que no existe relación estadística directa entre la variable bienestar subjetivo e ingreso económico absoluto. De igual manera, no existe relación significativa entre el bienestar objetivo y el subjetivo, salvo una correlación débil entre la tenencia de bienes materiales y los factores egocéntricos.

Finalmente, se encontró que los individuos empleados reportan niveles más elevados de bienestar subjetivo, en comparación con los desempleados. Estos resultados se discuten desde los principios del desarrollo humano, valorando la utilidad metodológica del bienestar subjetivo para la detección de desigualdades e indicador de calidad de vida.

Palabras claves: bienestar subjetivo, felicidad, satisfacción con la vida, bienestar objetivo, desarrollo humano

ABSTRACT: This research aims to analyze subjective well-being, in habitants of the urban municipality of Tatumbla, Francisco Morazán, Honduras, the research was made during the first semester of 2016. Particularly, subjective wellbeing is studied in relation to variables such as income, the objective well-being and employment status. All this was made through a quantitative approach, with a non-experimental design, and relational-transversal scope. Data for three subjective wellbeing dimensions (egocentric, homeostatic and sociocentric) were gather through a questionnaire.

The results indicate that there is no direct statistical relationship between subjective well-being and absolute income. However, the egocentric dimension is associated with relative income in the sociological and psychological standards. There is no significant relationship between objective and subjective well-being, except for a weak correlation between ownership of material goods and egocentric factors.

Finally, it was found that employed individuals report higher levels of subjective well-being, compared to unemployed. These results are discussed using human development principles as an analytic framework.

Keywords: Subjective well-being, happiness, satisfaction with life, objective well-being, human development

\section{Introducción}

La medición de variables económicas ha sido una estrategia ampliamente abordada para evaluar el desempeño nacional en cuanto a ingresos, producción y gastos. Tal es el caso de la cuantificación del Producto Interno Bruto (PIB), sin embargo, este indicador no integra aspectos fundamentales como los índices de alfabetismo, mortalidad infantil, expectativas de vida, entre otros (Stiglitz \& Walsh, 2005), ofreciendo una visión restringida del bienestar general de una sociedad. Por otro lado, entre los pilares que conforman el crecimiento económico se encuentran los recursos humanos de una población (Samuelson \& Nordhaus, 2010); el cual es representado por el tamaño de la fuerza laboral y su calidad en función a la educación, motivación y habilidades de los mismos.
Dicha visión del ser humano resulta reduccionista, limitando el valor de la persona a su capacidad para producir. Por lo tanto, el individuo se percibe como un medio de producción, en lugar de un fin en sí mismo. Como alternativa a dicho paradigma, se plantea la medición del desarrollo humano, el cual define los procesos para mejorar el bienestar de los individuos, ampliando sus libertades y oportunidades (Measure of America, 2015). Esto incluye aspectos socioeconómicos, legales, psicológicos, culturales, ambientales y políticos. De esta manera, se procura el fomento de condiciones necesarias e inclusivas que maximicen el potencial de los individuos. En este sentido, el desarrollo humano no se limita únicamente a la mejora de las condiciones relacionadas al ingreso y la riqueza material, sino que se centra en la riqueza de la vida humana (PNUD, 2016).

\footnotetext{
${ }^{1}$ Lic. en Psicología UNAH, Máster en Metodología de la Investigación UNAH, docente investigador de la Escuela de Psicología, facultad de Ciencias Sociales UNAH, correo electrónico mlandablanco @gmail.com
} 
La medición del desarrollo humano típicamente incluye indicadores respecto a la calidad de la experiencia humana en función a salud, educación y estándares dignos de vida. Para el año 2013, el Índice de Desarrollo Humano (IDH) de Honduras era de 0.617, clasificado como medio. En términos relativos, tal puntaje ubica al país en la posición 129 de 187 (PNUD, 2014). A pesar del carácter multidimensional del IDH, este aún no refleja otros aspectos de la experiencia humana. Evidenciando la necesidad de utilizar más indicadores que permitan tener valoración integral y amplia del desarrollo humano. Entre las dimensiones ausentes en los esquemas tradicionales del desarrollo humano, resalta el bienestar psicológico, la humillación, empoderamiento, calidad laboral y seguridad ciudadana (PNUD, 2016).

En este sentido, se han incorporado métricas que pretenden evaluar el bienestar desde lo subjetivo, tal es el caso de la medición de la felicidad. En tal temática, un referente ejemplar pertenece al Informe Mundial de Felicidad, el cual incluye un ranking de la misma, que considera los puntajes de satisfacción autoreportada con la vida, utilizando datos de la Encuesta Mundial de Gallup. Su aplicación se asiste de una escalera gráfica utilizada para que el encuestado enmarque su respuesta en cuanto a su grado de satisfacción con la vida y su grado de felicidad en la vida en general, cuantificado entre cero y diez (Helliwell, Layard, \& Sachs, 2016). De este informe se desprende el Ranking Mundial de la Felicidad, el cual ofrece una medición ordinal en donde se representan los puntajes de felicidad de 158 naciones, según los resultados obtenidos en la Encuesta Mundial de Gallup.

En el Ranking Mundial de la Felicidad 2012-2014, Honduras cuenta con un puntaje de 4.788 , ubicándolo en el puesto número 105 de 158 países (Helliwell, Huang, \& Wang, 2015). Dado el carácter subjetivo de la satisfacción con la vida, sus significados, dimensiones y determinantes, se aprecian en relación con las condiciones objetivas y culturales, la metodología utilizada para evaluar la felicidad de los hondureños es la misma que se utiliza para evaluar al resto de los habitantes del mundo. Esto a pesar de que las condiciones psicológicas, sociales y educativas, del contexto latinoamericano en general y hondureño en particular, son distintas al de países más desarrollados. En este contexto se evidencia la necesidad de validar instrumentos de medición del bienestar subjetivo que se acoplen a la realidad local.

Por otro lado, existen diversas fuentes de variación en la relación entre el ingreso y el bienestar, por lo cual este se considera un indicador limitado de la calidad de vida. Adicionalmente, la libertad de los individuos recae en su capacidad para elegir una vida que sea subjetivamente valiosa (Sen, 1999). Para ello es necesaria la conversión de bienes primarios en medios para el cumplimiento de fines personales.

El bienestar objetivo hace referencia a condiciones materiales y a la calidad de vida de las personas, esto incluye su ingreso económico, las condiciones de vivienda, oportunidades de educación, situaciones ambientales, seguridad, calidad del ambiente social y gozar de derechos civiles (Alatartseva \& Barysheva, 2015).

Considerando que las funcionalidades humanas reflejan aquello que el individuo valora ser o hacer, resulta fundamental estudiar el bienestar subjetivo, el cual se define como los juicios evaluativos, las construcciones cognitivas y afectivas, que un individuo realiza respecto a su vida (Cuadra \& Florenzano, 2003).

El presente artículo utiliza los términos felicidad, satisfacción con la vida y bienestar subjetivo de manera intercambiable. De esta forma, es posible complementar las medidas objetivas con las subjetivas, ofreciendo un panorama integral de las condiciones humanas. Los datos respecto a la satisfacción de necesidades materiales no son suficientes para comprender el bienestar subjetivo, por lo que se requiere adicionar información respecto a la satisfacción de necesidades psicológicas (Rojas, 2011).

Estudios realizados, han encontrado que existe una relación positiva entre la felicidad y el ingreso per cápita, sin embargo, la misma es moderada y no lineal. Por lo que una vez alcanzado determinado nivel de ingreso económico, la relación entre ambas variables tiende a aplanarse (Malcolm \& Fang, 2006).

Bajo tal consideración, la presente investigación tomó como foco de estudio el bienestar subjetivo como dimensión de desarrollo humano, en habitantes del casco urbano del municipio de Tatumbla, Francisco Morazán, Honduras. Para el 2009, se estima que el IDH de dicho municipio era de 0.661 (PNUD , 2012). Se contrastaron los resultados con variables como la condición laboral, ingreso económico absoluto (medido por medio de ingreso económico mensual, auto-reportado por el informante) y el bienestar objetivo (tenencia de bienes materiales, acceso a servicios, entre otros).

\section{Metodología}

\subsection{Diseño}

La presente investigación se enmarcó en un abordaje ba- 
sado en el enfoque cuantitativo, ya que estudió el fenómeno de manera numérica, con la utilización de herramientas estadísticas que permitieron analizar las variables de interés (Hernández, Fernández, \& Baptista, 2010).

El alcance fue correlacional, debido a que se estudió las relaciones entre el bienestar subjetivo e indicadores como el ingreso económico, bienestar objetivo, variables demográficas, entre otras. El tipo de investigación fue de corte transversal con un diseño no experimental, lo que indica que no existió manipulación de variables, ya que únicamente se observó el objeto de estudio en su ambiente natural, para ser posteriormente analizado (Hernández, Fernández, \& Baptista, 2010).

\subsection{Población y muestra}

El casco urbano de Tatumbla está compuesto por ocho barrios, habitados por 943 adultos. Sobre esta población, se utilizó la fórmula para determinar el tamaño de la muestra en el caso de medias, con universos finitos. Para calcular el tamaño de la muestra se tomó un margen de error de 1.25 y un nivel de confianza del $90 \%(z=1.645)$, con una desviación estandar de 12.45 , dando como resultado una muestra de 210 individuos. La distribución de tales unidades fue estratificada en base a sexo y barrio, con asignación proporcional al tamaño poblacional. La selección final de las unidades de análisis fue no probabilística, de tipo incidental.

\subsection{Técnicas de recolección de información}

Para la presente investigación, se diseñó y validó un instrumento de medición del Bienestar Subjetivo. El instrumento original estaba conformado por 36 reactivos en formato de Escala Likert, con tres opciones de respuesta que oscilaban entre "Nunca" (1 punto), "A veces" (2 puntos) y "Siempre" (3 puntos), se estima que mientras menor sea la complejidad de la respuesta, se requiere menor esfuerzo cognitivo para responder adecuadamente. Esta premisa parece particularmente pertinente al considerar el nivel educativo de las comunidades rurales de Honduras.

Posteriormente, se realizó una aplicación piloto en comunidades rurales, aledañas al casco urbano de Tatumbla. Con el propósito de tener una medición enmarcada en criterios científicos, se realizó una depuración de ítems en cuanto a los criterios de confiabilidad y validez. Para simplificar la estructura dimensional de la escala, se realizó una reducción de factores. Además, se obtuvieron medidas de consistencia interna como el Alfa de Cronbach y la división por mitades. Se calculó la validez convergente de la escala depurada, asegurando así que en efecto el instrumento mida la variable de estudio.
Se utilizó el programa SPSS para reducir los ítems a factores, se realizó la agrupación mediante el Método de Extracción de Componentes Principales, extrayendo auto-valores mayores a 2. Adicionalmente, se ejecutó rotación de componentes utilizando el Método Quartimax, con normalización Kaiser. Se suprimieron los coeficientes que tuviesen un valor absoluto menor a .6, la combinación de tales especificaciones logró la reducción de los ítems a tres factores. Estos son: factores egocéntricos $(\alpha=.854)$, sociocéntricos $(\alpha=.778)$ y homeostáticos $(\alpha=.834)$, los cuales conforman la escala total $(\alpha=.877)$.

Para obtener una medida de consistencia interna de la escala total, se obtuvo un coeficiente de confiabilidad por división por mitades. Como criterio de división, se tomaron los reactivos de la primera mitad de la escala, correlacionados contra los ítems de la segunda mitad del instrumento. Esto resulta en un puntaje corregido de Spearman-Brown de .751, por lo cual se considera que la escala tiene una consistencia interna aceptable. Al correlacionarlo con el Índice de Bienestar Personal, versión para Adultos, Quinta Edición (International Wellbeing Group, 2013), se obtuvo un coeficiente de validez convergente de .767 .

A continuación, se presentan algunos ejemplos de los ítems que componen la escala final. Para el factor egocéntrico: "soy una persona feliz", "me siento satisfecho(a) con mi salud", "me gusta mi vida", "me gusta cómo es mi cuerpo", entre otros. En el factor sociocéntrico: "siento que soy importante para mi familia", "me siento satisfecho(a) con mis vecinos", "la gente me respeta", etc. Finalmente, la dimensión homeostática: "estoy satisfecho(a) con mi vida espiritual", "me siento seguro en mi comunidad", "me siento seguro dentro de mi casa", "me siento satisfecho con cuánto dinero tengo", etc.

\section{Discusión de resultados}

\subsection{Descripción general de componentes}

Los reactivos agrupados para el factor egocéntrico hacen alusión a percepciones acerca del propio ser, puntualmente aborda indicadores vivenciales y corporales y la relevancia que el individuo tiene para sí mismo (Landa Blanco \& Landa Blanco, 2016). La dimensión sociocéntrica conglomera reactivos que expresan la satisfacción en cuanto a sujetos externos, o bien la interacción del individuo con tales personas, esto incluye familiares, amigos, vecinos. De igual manera, se manifiesta una percepción de valía dictada desde las estructuras sociales externas, tal es el caso de la noción de ser importante para otro, así

Revista Población y Desarrollo: argonautas y caminantes 
como de ser respetado. Finalmente, los ítems asociados al factor homeostático indican un sentido de estabilidad y protección en cuanto a lo espiritual, físico, infraestructural y económico.

\subsection{Relación entre bienestar subjetivo e ingreso económico absoluto}

Para indagar la relación entre Bienestar Subjetivo e Ingreso Económico Absoluto, se utilizó como estadígrafo la rho de Spearman. Los resultados para la escala total obtuvieron una rho $(215)=.042, p=.544$. Indicando que no existe relación estadísticamente significativa entre el ingreso económico absoluto y el bienestar subjetivo. Lo misma decisión resulta al analizar cada una de las dimensiones contra el ingreso, Factores Egocéntricos rho $(215)=.078, p=.252$, Factores Sociocéntricos rho (215) $=-.065, p=.340$ y Factores Homeostáticos rho $(215)=.038, p=.581$, para un resumen de tales datos ver Cuadro $\mathrm{N}^{\circ} 1$.

Cuadro $N^{\circ} 1$. Relación entre el bienestar subjetivo e ingreso económico absoluto

\begin{tabular}{|l|l|l|l|l|l|}
\hline \multicolumn{2}{|l|}{ Elementos } & Correlación & Sig. & $\begin{array}{l}\text { Hipótesis } \\
\text { Nula }\end{array}$ & Decisión \\
\hline \multirow{3}{*}{$\begin{array}{l}\text { Ingreso } \\
\text { económico } \\
\text { absoluto }\end{array}$} & Egocéntricos & 0.078 & 0.252 & & No rechazar \\
\cline { 2 - 4 } \cline { 3 - 4 } & Sociocéntricos & -0.065 & 0.34 & \multirow{2}{*}{ rho=0 } & No rechazar \\
\cline { 2 - 4 } \cline { 3 - 4 } & Homeostáticos & 0.038 & 0.581 & & No rechazar \\
\cline { 2 - 4 } & Escala Total & 0.042 & 0.544 & & No rechazar \\
\hline
\end{tabular}

Fuente: Elaboración propia

Estos resultados pueden ser analizados desde los argumentos que plantean que una vez satisfechas las necesidades básicas, las personas tienden a adaptarse a su nivel económico. En este punto, el bienestar subjetivo de los individuos no se ve significativamente influenciado por sus ingresos económicos (Cuadra \& Florenzano, 2003). Esto resalta el hecho de que generalmente la satisfacción con la vida se mantiene constante a pesar de los incrementos en el ingreso (Weimann, Knabe, \& Schob, 2015).

Para una mejor comprensión de la dinámica entre ambas variables se ejecutaron correlaciones ítem-ingreso económico absoluto. No obstante, el único coeficiente estadísticamente significativo corresponde a la relación entre el ítem "Tengo suficiente comida" y el ingreso económico absoluto, rho (215) $=.262, p=.000$. Indicando que, a mayor ingreso económico absoluto, mayor satisfacción en cuanto a la tenencia de comida, pero, a pesar de su significancia inferencial, dicho coeficiente se clasifica como débil. Se debe considerar que, durante el periodo incluido entre marzo y mayo del año 2016 , el costo de la canasta básica era de Lps 5,444.30, para la zona de Distrito Central (Consejo Hondureño de la Empresa Privada, 2016). Se calculó el ingreso promedio de los encuestados utilizando medidas descriptivas para datos agrupados, los encuestados manifiestan tener un ingreso promedio de Lps 7,615.46, con una desviación estándar de Lps. $8,636.21$. Dicha información podría contextualizar la relación encontrada entre ingreso económico absoluto y satisfacción con cuanta comida se tiene. Además, resalta que no existe correlación estadísticamente significativa entre el ingreso económico absoluto y el reactivo "Me siento satisfecho con cuánto dinero tengo", rho (215) $=.051, p=.455$.

El presente resultado parece indicar que la satisfacción obedece a una dinámica psicológica interna, más que dependiente de los estímulos externamente disponibles, reflejando que la satisfacción con cuanto ingreso económico se tenga, no se relaciona directamente con el ingreso económico absoluto. A pesar de ello, no se descarta el carácter mediador que puede tener el ingreso sobre otros indicadores, como la disponibilidad de alimentos; pudiendo servir como factor protector del bienestar subjetivo, en contra de las fallas homeostáticas.

\subsection{Relación entre bienestar subjetivo y bienestar objetivo}

Para poder evaluar el bienestar objetivo de los individuos, se les preguntó acerca de la tenencia de distintos bienes, materiales de construcción de la vivienda, acceso a servicios básicos, manejo de excretas, medios de transporte. A partir de estos datos, se construyó el Índice de Bienestar Objetivo, el cual obtuvo un Coeficiente de Correlación de Spearman, estadísticamente significativo, rho $(217)=.634, p=.000$, con el ingreso económico de los individuos, este coeficiente se clasifica como moderadamente fuerte.

Los resultados indican que no existe relación estadísticamente significativa entre el bienestar subjetivo y el Índice de Bienestar Objetivo, rho (217) $=.055$, $p=.418$. Esta tendencia se mantiene para cada una de las dimensiones que conforman la escala de bienestar subjetivo, ver cuadro $\mathrm{N}^{\circ} 2$. En análisis por ítem, reporta que existe correlación positiva estadísticamente significativa entre el reactivo "Tengo suficiente comida" y el Índice de Bienestar Objetivo, rho (217) =.334, $p=.000$. Esto sugiere que los individuos que reportan elevados niveles de satisfacción con la disponibilidad de alimentos, también tienden a reportar mayores niveles de bienestar objetivo. 
Cuadro $N^{\circ}$ 2. Relación entre bienestar subjetivo y bienestar objetivo

\begin{tabular}{|c|l|c|c|c|l|}
\hline \multicolumn{2}{|c|}{ Elementos } & $\begin{array}{c}\text { Correla- } \\
\text { ción }\end{array}$ & Sig. & $\begin{array}{c}\text { Hipóte- } \\
\text { sis Nula }\end{array}$ & \multicolumn{1}{|c|}{ Decisión } \\
\hline \multirow{4}{*}{ Bienestar Objetivo } & Egocéntricos & .099 & .146 & & No rechazar \\
\cline { 2 - 4 } & Sociocéntricos & -.023 & .736 & \multirow{4}{*}{ rho=0 } & No rechazar \\
\cline { 2 - 3 } & Homeostáticos & .004 & .955 & & No rechazar \\
\cline { 2 - 3 } & Escala Total & .055 & .418 & & No rechazar \\
\hline
\end{tabular}

Fuente: Elaboración propia

Resalta que, en el caso de los hombres, existe una relación negativa débil estadísticamente significativa entre el Índice de Bienestar Objetivo y el reactivo "Me siento satisfecho con mis vecinos", rho $(107)=-.200, p=.039$. Lo que indica débilmente que, a mayor bienestar objetivo, menor satisfacción con los vecinos. Tales resultados pueden ser explicados desde las normativas de comparación relativa sociológicas. En este sentido, es posible que los individuos que posean mayor bienestar objetivo, consideren que existe una marcada diferencia social con sus vecinos inmediatos, disminuyendo la satisfacción con los mismos.

Paralelamente, el ítem "Me gusta mi casa" se correlaciona débilmente con cuan satisfecho se encuentra el individuo con sus ingresos económicos, rho $(217)=.298, p=.000$. Resalta que ambos indicadores reflejan valoraciones exclusivamente subjetivas, ya que, al comparar el grado de satisfacción hacia la vivienda, con un indicador objetivo como el ingreso económico, no existe relación significativa entre ambas variables rho $(215)=-.068, p=.322$. Tal dinámica persiste al evaluar el grado de asociación entre la satisfacción con la vivienda y el Índice de Bienestar Material, con el cual tampoco existe relación rho (216) $=-.054, p=.425$. En este sentido, se evidencia la relevancia de las mismas variables subjetivas en la explicación de la satisfacción con la vida.

Para obtener una comprensión más detallada de la dinámica entre el bienestar subjetivo y la tenencia de bienes materiales, se construyó un índice de estos últimos. A diferencia del Índice de Bienestar Objetivo, el Índice de Bienes Materiales, se limita a considerar la tenencia de bienes como sillas, teléfono celular, computadora, ventilador, motocicleta, automóvil, etc., excluyendo acceso a servicios básicos y materiales de construcción de la vivienda. En este sentido, se encontró relación positiva débil, pero estadísticamente significativa, entre los puntajes obtenidos en la escala total y la tenencia de bienes materiales, rho $(217)=.146, p=.031$. Según lo expuesto en el Cuadro $\mathrm{N}^{\circ} 3$, esta dinámica no aplica para los factores Sociocéntricos y Homeostáticos, pero si para la dimensión Egocéntrica. Lo que indica que los individuos que reportan mayor satisfacción con argumentos orientados al yo, como "soy una persona feliz" o "tengo suficiente comida", también poseen una mayor cantidad de bienes materiales.

\section{Cuadro $\mathrm{N}^{\circ} 3$. Bienestar subjetivo y tenencia de bienes materiales}

\begin{tabular}{|l|l|l|l|l|l|}
\hline \multicolumn{2}{|l|}{ Elementos } & Correlación & Sig. & $\begin{array}{l}\text { H i pó t e s i s } \\
\text { Nula }\end{array}$ & Decisión \\
\hline \multirow{4}{*}{ Tenenciade } & Egocéntricos & .197 & .004 & & Rechazar \\
\cline { 2 - 4 } & Sociocéntricos & .030 & .662 & \multirow{2}{*}{ rho=0 } & No rechazar \\
\cline { 2 - 4 } & Homeostáticos & .041 & .548 & & No rechazar \\
\cline { 2 - 4 } & Escala Total & .146 & .031 & & Rechazar \\
\hline
\end{tabular}

Fuente: Elaboración propia

\subsection{Bienestar subjetivo y condición laboral}

De los individuos encuestados, el $65.4 \%$ reporta estar empleado, mientras que el restante $34.6 \%$ corresponde a la población desempleada. Para probar la diferencia en el Bienestar Subjetivo en función a la condición laboral, se utilizó la prueba no paramétrica $U$ de Mann-Whitney. El Gráfico $N^{\circ} 1$ muestra la distribución de tales puntajes.

\section{Gráfico $N^{\circ} 1$. Distribución del bienestar subjetivo, en función a la condición laboral}

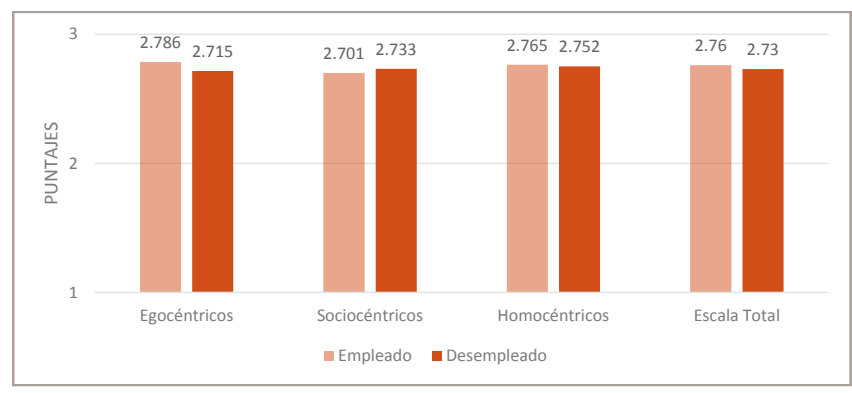

Fuente: Elaboración propia

Los resultados indican que no existe diferencia estadísticamente significativa entre los puntajes obtenidos en la escala total, en función a la condición laboral. Lo mismo aplica para las dimensiones sociocéntricas y homeostáticas (Cuadro $\mathrm{N}^{\circ} 4$ ). No obstante, el factor egocéntrico se muestra significativamente más elevado para los individuos empleados, en comparación con los desempleados.

El análisis por reactivos indica que la satisfacción con el reactivo "Tengo suficiente comida", es significativamente más alto $(\mathrm{p}=.007)$ para los empleados $(\overline{\mathrm{x}}=2.845, \mathrm{~s}=.382)$, que para los desempleados $(\bar{x}=2.693, s=.464)$. De manera simplista, se podría esperar que el acceso al mercado 
Cuadro $N^{\circ} 4$. Resumen de prueba de hipótesis bienestar subjetivo, en función a la condición laboral

\begin{tabular}{|c|c|c|c|c|c|}
\hline Factor & Agrupación & Hipótesis Nula & Prueba & Sig. & Decisión \\
\hline & \multirow{4}{*}{$\begin{array}{l}\text { Condición } \\
\text { laboral }\end{array}$} & \multirow{4}{*}{$\begin{array}{l}\text { Bienestar } \\
\text { Subjetivo } \\
\text { Empleado= } \\
\text { Bienestar } \\
\text { Subjetivo } \\
\text { Desempleado }\end{array}$} & \multirow{4}{*}{$\begin{array}{l}\text { U de } \\
\text { Mann-Whit- } \\
\text { ney de } \\
\text { muestras } \\
\text { indepen- } \\
\text { dientes }\end{array}$} & 0.017 & $\begin{array}{l}\text { Rechazar la } \\
\text { hipótesis nula }\end{array}$ \\
\hline $\begin{array}{l}\text { Sociocéntrico } \\
\text { Egocéntrico }\end{array}$ & & & & 0.283 & $\begin{array}{l}\text { No rechazar la } \\
\text { hipótesis nula }\end{array}$ \\
\hline Homeostático & & & & 0.872 & $\begin{array}{l}\text { No rechazar la } \\
\text { hipótesis nula }\end{array}$ \\
\hline Escala Total & & & & 0.272 & $\begin{array}{l}\text { No rechazar la } \\
\text { hipótesis nula }\end{array}$ \\
\hline
\end{tabular}

Fuente: Elaboración propia

laboral implique una retribución económica, con la cual se mejora el acceso al consumo alimentario. Congruentemente, la falta de empleo representaría una disminución en la capacidad económica necesaria para acceder dignamente a los productos de la canasta básica. En este sentido, los encuestados que reportan estar empleados, muestran un ingreso familiar mensual promedio de $9,067.20$ lempiras, mientras que el de los desempleados es de 4,866.83 lempiras (ingreso obtenido por medio de rentas, pensiones, remesas, etc.)

Por otro lado, los puntajes para el ítem "Me gusta mi vida", también se muestran significativamente $(p=.041)$ más altos para los empleados ( $\bar{x}=2.908, s=.289$ ), que para los desempleados $(\bar{x}=2.8, s=.435)$. Se debe considerar que el trabajo no se limita a la ejecución de tareas, sino que además tiene implicaciones sociales y psicológicas que pueden fomentar el desarrollo humano.

\section{Reflexión final}

En conjunto, los resultados obtenidos resaltan la viabilidad y relevancia de la medición del bienestar subjetivo como dimensión de desarrollo humano local. Mostrándose particularmente útil en la evaluación de las condiciones sociales que envuelven a los individuos. No obstante, la ausente relación entre el bienestar subjetivo e indicadores económicos, así como la débil relación con las condiciones materiales, conlleva a un replanteamiento de las nociones tradicionales respecto a la acumulación de bienes como motivador de la felicidad. A pesar de ello, es innegable la necesidad de fomentar el bienestar objetivo, ya que la adaptación subjetiva a la precariedad de condiciones materiales y económicas, de ninguna manera justifica la consolidación de las mismas, indicando el carácter complementario, no sustitutivo, del bienestar subjetivo-objetivo, como reflejo de desarrollo humano.

La necesidad de considerar el desarrollo humano, como un fenómeno holístico, se evidencia en el último informe regional para Centroamérica y El Caribe, titulado Progreso Multidimensional: Bienestar más allá del ingreso (PNUD, 2016). En este sentido, dado el carácter multivariado de las necesidades y aspiraciones humanas, el mero crecimiento económico es insuficiente para lograr el desarroIlo. De esta manera, se admite la necesidad de integrar nuevas métricas que ofrezcan un panorama más amplio respecto a las mediciones multidimensionales, como el bienestar psicológico. Las implicaciones de esto, promoverían una evolución teórica y metodológica que permita trascender de un abordaje de la persona como concepto deficitario (centrado en carencias) a un abordaje del bienestar, enfocado en el ser y hacer.

\section{Bibliografía}

A Alatartseva, E., \& Barysheva, G. (2015). Well-being: subjective and objective aspects. Social and Behavioral Sciences, 36 - 42. doi: 10.1016/j.sbspro.2014.12.479

M Consejo Hondureño de la Empresa Privada. (2016). Boletín Semanal Canasta Básica de Alimentos. Centro de Investigaciones Económicas y Sociales, Tegucigalpa.

M Cuadra, H., \& Florenzano, R. (2003). El Bienestar Subjetivo: Hacia una Psicología Positiva . Revista de Psicología de la Universidad de Chile , 83-96.

Helliwell, J., Huang, H., \& Wang, S. (2015). The Geography of World Happiness. In J. Helliwell, R. Layard, \& J. Sachs (Eds.), World Happiness Report 2015 (pp. 26-28). Sustainable Development Solutions Network . Retrieved from http://worldhappiness.report/wp content/uploads/ sites/2/2015/04/WHR15.pdf

W Helliwell, J., Layard, R., \& Sachs, J. (2016). Happiness Report 2016 Update (Volume I). New York: Sustainable Development Solutions Network.

Mernández, R., Fernández, C., \& Baptista, P. (2010). Metodología de la Investigación. Peru:

oMcGraw-Hill. International Wellbeing Group. (2013). Personal Wellbeing Index: 5th Edition. Melbourne: Centre on Quality of Life, Deakin University.

Landa Blanco, M., \& Landa Blanco, A. (2016). Signifcados y dimensiones del bienestar subjetivo, en mujeres que habitan en la Aldea La Calera, Honduras. Economía y Administración, 7(2), 151 162. Obtenido de http://www. iies-unah.org/Revista/index.php/EyA/article/view/414

Malcolm, J., \& Fang, C. (2006). Homeostasis and Well 
Being. Singapore Management University. Singapore: Research Collection School Of Economics.

Measure of America. (2015). American Human Development Report: The Measure of America 2013-2014. Brooklyn: Measure of America.

M PNUD . (2012). Informe sobre Desarrollo Humano Honduras 2011. Honduras: Litografía e Imprenta Lil.

DNUD. (2014). Resumen Informe Sobre Desarrollo Humano 2014 Sostener el Progreso Humano: reducir vulnerabilidades y construir resiliencia. New York: Programa de las Naciones Unidas para el Desarrollo.

M PNUD. (2016). Informe Regional sobre Desarrollo Humano para América Latina y el Caribe:

Progreso multidimensional bienestar más allá del ingreso. New York.

Rojas, M. (2011). El bienestar subjetivo: su contribución a la apreciación y la consecución del progreso y el bienestar humano. Revista Realidad, Datos y Espacio. Revista Internacional de Estadística y Geografía, 2(1), 64-77.

M Samuelson, P., \& Nordhaus, W. (2010). Economía con aplicaciones a Latinoamérica. México D.F.: McGraw-Hill E.

Sen, A. (1999). Development as Freedom. New York: Random House.

Stiglitz, J., \& Walsh, C. (2005). Economics. New York : W. W. Norton \& Company.

Weimann, J., Knabe, A., \& Schob, R. (2015). Measuring Happiness: The economics of well-being.Massachusetts: Massachusetts Institute of Technology. 